\title{
The Quasi-Static Approximation of Heat Waves in Anisotropic Thermo-Elastic Media
}

\author{
Shaohua Guo \\ School of Civil Engineering and Architecture, Zhejiang University of Science and Technology, \\ Hangzhou, China \\ E-mail: gsh606@yahoo.com.cn \\ Received August 6, 2010; revised September 21, 2010; accepted September 25, 2010
}

\begin{abstract}
The equilibrium equations of anisotropic media, coupled to the heat conduction equations, are studied here based on the standard spaces of the physical presentation, in which an new thermo-elastic model based on the second law of thermodynamics is induced. The uncoupled heat wave equation for anisotropic media is deduced. The results show that the equation of heat wave is of the properties of dissipative waves. In final part of this paper, we discuss the propagation behaviour of heat waves for transversely isotropic media.
\end{abstract}

Keywords: Anisotropic Media, Thermo-Elastic Model, Heat Wave, Standard Spaces, Modal Equations

\section{Introduction}

In recent years, the considerable interests have been shown in the study of thermo-elastic wave propagations in anisotropic media. The classical theory of thermoelasticity is based on Fourier's law of heat conduction, which predicts an infinite speed of propagation of heat. This is physically absurd and some new theories have been proposed to eliminate this absurdity. For example, Lord and Shulman [1] obtained a wave-type heat equation by modifying the Fourier's law of heat conduction. This new law contains the heat flux vector as well as its time derivative. It also contains a new constant that acts as a relaxation time. Since the heat equation of this theory is of the wave type, it automatically ensures finite speeds of propagation for heat and elastic waves. The remaining governing equations for this theory, namely, the equations of motions and constitutive relations, remain the same as those for the coupled and the uncoupled theories. This theory was extended by Dhaliwal and Sherief [2] to general anisotropic media in the presence of heat sources. Later, Green and Lindsay [3] deduced another theory, known as temperature rate dependent theory of thermo-elasticity, including the rate of temperature in the constitutive equations. This theory contains two constants that act as relaxation times and modifies all the equations of the coupled theory, not only the heat equation. The classical Fourier's law of heat conduction is not violated if the medium under consider- ation has a center of symmetry. Although many works have been done for heat or elastic waves in anisotropic media, the explicit uncoupled equations of wave equations in anisotropic media could not be obtained because of the limitations of classical elastic theory [4-6]. In this paper, the idea of standard spaces [7-10] is used to deal with both the equilibrium equation and the heat conduction equation based on the quasi-static approximation of the propagation of heat wave, and by introducing an new thermo-elastic model that obeys the second law of thermo-dynamics, a uncoupled modal equations of heat wave are obtained, which shows that heat wave is of the properties of dissipative waves. Meanwhile the propagation speed, propagation direction and space pattern of heat wave can be completely determined by the modal equations.

\section{Modal Constitutive Equation of Anisotropic Thermo-Elastic Media}

Hooke's law and entropy relationship for linear thermoelastic materials are the following

$$
\begin{gathered}
\sigma_{i j}=c_{i j k l} \varepsilon_{k l}-a_{i j} \theta \\
\rho \eta=a_{k l} \varepsilon_{k l}+v \theta
\end{gathered}
$$

Rewriting them in the Voigt's notation, we have

$$
\begin{gathered}
\boldsymbol{\sigma}=\boldsymbol{c} \cdot \boldsymbol{S}-\boldsymbol{a} \cdot \theta \\
\rho \eta=\boldsymbol{a}^{T} \cdot \boldsymbol{S}+\boldsymbol{v} \cdot \theta
\end{gathered}
$$


The elastic matrix $\boldsymbol{c}$ can be spectrally decomposed as follows ${ }^{[7-10]}$

$$
\boldsymbol{c}=\boldsymbol{\Phi} \boldsymbol{\Lambda} \boldsymbol{\Phi}^{\mathrm{T}}
$$

where $\boldsymbol{\Lambda}=\operatorname{diag}\left[\lambda_{1}, \lambda_{2}, \lambda_{3}, \lambda_{4}, \lambda_{5}, \lambda_{6}\right]$, , are the matrixes of eigen elasticity. $\Phi=\left\{\boldsymbol{\varphi}_{1}, \boldsymbol{\varphi}_{2}, \boldsymbol{\varphi}_{3}, \boldsymbol{\varphi}_{4}, \boldsymbol{\varphi}_{5}, \boldsymbol{\varphi}_{6}\right\}$ is the modal matrix of elastic media, which is both orthogonal and positive definite matrixes, and satisfy $\boldsymbol{\Phi}^{\mathrm{T}} \boldsymbol{\Phi}=\boldsymbol{I}$ 。

Projecting the elastic physical qualities of the geometric presentation, such as the stress vector $\sigma$ and strain vector $\boldsymbol{S}$, into the standard spaces of the physical presentation, we get

$$
\begin{aligned}
\boldsymbol{\sigma}^{*} & =\boldsymbol{\Phi}^{\mathrm{T}} \cdot \boldsymbol{\sigma} \\
\boldsymbol{S}^{*} & =\boldsymbol{\Phi}^{\mathrm{T}} \cdot \boldsymbol{S}
\end{aligned}
$$

Rewriting Equations (6) and (7) in the form of scalar, we have

$$
\begin{aligned}
& \sigma_{i}^{*}=\boldsymbol{\varphi}_{i}^{* \mathrm{~T}} \cdot \boldsymbol{\sigma} \quad i=1, \cdots, m \\
& S_{i}^{*}=\boldsymbol{\varphi}_{i}^{* \mathrm{~T}} \cdot \boldsymbol{S} \quad i=1, \cdots, m
\end{aligned}
$$

where $m(\leq 6)$ is number of the elastic independent subspaces. Equations (8) and (9) show the elastic physical qualities under the physical presentation.

Substituting Equations (6) and (7) into Equations (3) and (4), respectively, and multiplying them with the transpose of modal matrix in the left, we have

$$
\begin{aligned}
\boldsymbol{\Phi}^{\mathrm{T}} \boldsymbol{\sigma} & =\boldsymbol{\Phi}^{\mathrm{T}} \boldsymbol{c} \boldsymbol{\Phi} \boldsymbol{S}^{*}-\boldsymbol{\Phi}^{\mathrm{T}} \boldsymbol{a} \theta \\
\rho \eta & =\boldsymbol{a}^{T} \boldsymbol{\Phi} \boldsymbol{S}^{*}+v \cdot \theta
\end{aligned}
$$

Using Equations (5), (6) and (7), we get

$$
\begin{aligned}
& \sigma^{*}=\Lambda S^{*}-a^{*} \theta \\
& \rho \eta=a^{* T} \cdot S^{*}+v \theta
\end{aligned}
$$

Rewriting the above equations in the form of scalar, we have

$$
\begin{gathered}
\sigma_{i}^{*}=\lambda_{i} S_{i}^{*}-a_{i}^{*} \theta \quad i=1-m \\
\rho \eta=a_{k}^{*} S_{k}^{*}+v \theta \quad k=1-m \quad \text { sum to k }
\end{gathered}
$$

Equations (14) and (15) are just the modal constitutive equations for anisotropic thermo-elastic media, in which $a_{k}^{*}$ are the coupled thermo-elastic coefficients.

\section{Heat Conduction and Thermo-Dynamic Equations}

The Fourier's law of heat conduction is the following

$$
q_{i}=-\beta_{i j} \theta_{i j}
$$

where $\theta=T-T_{0}$, The second law of thermo-dynamics is the following

$$
d s=d s_{e}+d s_{i}
$$

where $d s_{e}=\frac{d Q}{T}, d s \geq 0$ and $d s_{i} \geq 0$. To general anisotropic media in the absence of heat sources, Equation (17) can be written in terms of entropy density as follows

$$
T \rho \dot{\eta}=-q_{i^{\prime} i}+T \rho \dot{\eta}_{i}
$$

Now, we suppose that the irreversible part of entropy density rate is direct proportion to the negative value of entropy density acceleration

$$
\dot{\eta}_{i}=-\tau d \ddot{\eta}
$$

The reason for this is that $d \eta_{i} \geq 0, d \dot{\eta} \leq 0$, which are requirements to maintain a stable thermodynamic process. Then, by Equation (18), we have

$$
q_{i^{\prime} i}=-T \rho\left(1+\tau \nabla_{t}\right) \dot{\eta}
$$

Differentiating Equation (16) with respect to $i$ and substituting from Equation (20), we get

$$
\beta_{i j} \theta_{{ }_{j i}}=T \rho\left(1+\tau \nabla_{t}\right) \nabla_{t} \eta
$$

This is the thermodynamic equation based on the second law of thermodynamics, which is same as the L-S model [1].

\section{Eigen Expression of Equilibrium and Thermodynamic Equation}

The eigen form of elastic equilibrium equation can be written as follows $[7,8]$

$$
\Delta_{i}^{*} \sigma_{i}^{*}=0
$$

where, $\Delta_{i}^{*}=\left\{\varphi_{i}^{*}\right\}^{T}[\Delta]\left\{\varphi_{i}^{*}\right\}$ is the stress operator $[7,8]$, in which

$$
[\Delta]=\left[\begin{array}{cccccc}
\partial_{11} & 0 & 0 & 0 & \partial_{31} & \partial_{21} \\
0 & \partial_{22} & 0 & \partial_{32} & 0 & \partial_{21} \\
0 & 0 & \partial_{33} & \partial_{32} & \partial_{31} & 0 \\
0 & \partial_{23} & \partial_{23} & \left(\partial_{22}+\partial_{33}\right) & \partial_{21} & \partial_{31} \\
\partial_{13} & 0 & \partial_{13} & \partial_{12} & \left(\partial_{11}+\partial_{33}\right) & \partial_{32} \\
\partial_{12} & \partial_{12} & 0 & \partial_{13} & \partial_{23} & \left(\partial_{22}+\partial_{11}\right)
\end{array}\right]
$$

We can also rewrite Equation (21) in form of matrix as follows

$$
\{B\}^{T}\{\nabla\} \theta=T \rho\left(1+\tau \nabla_{t}\right) \nabla_{t} \eta
$$

where

$$
\begin{aligned}
& \{B\}^{T}=\left\{\beta_{11}, \beta_{22}, \beta_{33}, 2 \beta_{23}, 2 \beta_{31}, 2 \beta_{12}\right\} \\
& \{\nabla\}^{T}=\left\{\nabla_{11}, \nabla_{22}, \nabla_{33}, \nabla_{23}, \nabla_{31}, \nabla_{12}\right\} .
\end{aligned}
$$

Let $\nabla^{*}=\{B\}^{T}\{\nabla\}$, Equation (24) becomes 


$$
\nabla^{*} \theta=T \rho\left(1+\tau \nabla_{t}\right) \nabla_{t} \eta
$$

\section{Modal Equation of Heat Wave in Anisotropic Media}

Substituting Equations $(14,15)$ into Equations (22) and (25), we have

$$
\begin{gathered}
\Delta_{i}^{*}\left[\lambda_{i} S_{i}^{*}-a_{i}^{*} \theta\right]=0 \\
\nabla^{*} \theta=T\left(1+\tau \nabla_{t}\right) \nabla_{t}\left(a_{k}^{*} S_{k}^{*}+v \theta\right)
\end{gathered}
$$

According to the principle of operator, Equation (26) can be written as follows

$$
S_{k}^{*}=\frac{a_{k}^{*}}{\lambda_{k}} \theta
$$

By using Equation (28), Equation (27) becomes

$$
\nabla^{*} \theta-\left(v+\sum_{k} \frac{a_{k}^{*} a_{k}^{*}}{\lambda_{k}}\right) T \nabla_{t} \theta-\left(v+\sum_{k} \frac{a_{k}^{*} a_{k}^{*}}{\lambda_{k}}\right) T \tau \nabla_{t t} \theta=0
$$

Rewriting Equation (29) in the form of wave equation, we get

$$
\nabla_{t t} \theta+\frac{1}{\tau} \nabla_{t} \theta=c^{2} \nabla^{*} \theta
$$

where

$$
c=\sqrt{\frac{1}{\left(v+\sum_{k} \frac{a_{k}^{*} a_{k}^{*}}{\lambda_{k}}\right) T \tau}}
$$

It just the speed of heat wave in anisotropic media.

\section{Application}

In this section, we discuss the propagation laws of heat wave in a hexagonal (transversely isotropic) crystal. The material tensors in Equations (1), (2), (16) are represented by the bottom matrices (32) under the compact notation where $c_{66}=\frac{1}{2}\left(c_{11}-c_{12}\right)$.

There are four independent eigenspaces in a hexagonal (transversely isotropic) crystal [7-10]

$$
W=W_{1}^{(1)}\left[\varphi_{1}\right] \oplus W_{2}^{(1)}\left[\varphi_{2}\right] \oplus W_{3}^{(2)}\left[\varphi_{3}, \varphi_{6}\right] \oplus W_{4}^{(2)}\left[\varphi_{4}, \varphi_{5}\right]
$$

where as shown in Equation (34)

where $\xi_{i}$ is a vector of order 6 in which $i$ th element is 1 and others are 0 .

The eigenelasticity of hexagonal crystal are

$$
\left.\begin{array}{l}
\lambda_{1,2}=\frac{c_{11}+c_{12}+c_{33}}{2} \pm \sqrt{\left(\frac{c_{11}+c_{12}+c_{33}}{2}\right)^{2}+2 c_{13}^{2}}, \\
\lambda_{3}=c_{11}-c_{12}, \quad \lambda_{4}=c_{44}
\end{array}\right\}
$$

The structures of four independent eigen-spaces are the following

$$
\begin{aligned}
\varphi_{1,2}^{*} & =\varphi_{1,2}, \\
\varphi_{3}^{*} & =\frac{\sqrt{3}}{3}[1,-1,0,0,0,1]^{T}, \varphi_{4}^{*}=\frac{\sqrt{2}}{2}[0,0,0,1,1,0]^{T} \\
a_{1,2}^{*} & =\alpha_{1,2}\left[a_{11}+a_{22}+\gamma_{1,2} a_{33}\right],
\end{aligned}
$$

The relative quantities and operators can be calculated as follows:

$$
\begin{aligned}
& {\left[\begin{array}{llllll}
c_{11} & c_{12} & c_{13} & 0 & 0 & 0 \\
c_{12} & c_{11} & c_{13} & 0 & 0 & 0 \\
c_{13} & c_{13} & c_{13} & 0 & 0 & 0 \\
0 & 0 & 0 & c_{44} & 0 & 0 \\
0 & 0 & & & c_{44} & 0 \\
0 & 0 & 0 & 0 & 0 & c_{66}
\end{array}\right],\left[\begin{array}{lll}
a_{11} & 0 & 0 \\
0 & a_{11} & 0 \\
0 & 0 & a_{33}
\end{array}\right],\left[\begin{array}{lll}
\beta_{11} & 0 & 0 \\
0 & \beta_{11} & 0 \\
0 & 0 & \beta_{33}
\end{array}\right]} \\
& \left.\varphi_{1,2}=\frac{c_{13}}{\sqrt{\left(\lambda_{1,2}-c_{11}-c_{12}\right)^{2}+2 c_{13}^{2}}} \times\left[1,1, \frac{\lambda_{1,2}-c_{11}-c_{12}}{c_{3}}, 0,0,0\right]^{\mathrm{T}}\right] \\
& \varphi_{3}=\frac{\sqrt{2}}{2}[1,-1,0,0,0,0]^{\mathrm{T}} \\
& \varphi_{i}=\zeta_{i}, i=4,5,6
\end{aligned}
$$




$$
\begin{aligned}
& a_{3}{ }^{*}=\frac{\sqrt{3}}{3}\left(a_{11}-a_{22}\right), \\
& a_{4}{ }^{*}=0 \\
& \nabla^{*}=\beta_{11}\left(\partial_{x}^{2}+\partial_{y}^{2}\right)+\beta_{33} \partial_{z}^{2}
\end{aligned}
$$

where

$$
\alpha_{1,2}=\frac{c_{13}}{\sqrt{\left(\lambda_{1,2}-c_{11}-c_{12}\right)^{2}+2{c_{13}{ }^{2}}^{2}}}, \quad \gamma_{1,2}=\frac{\lambda_{1,2}-c_{11}-c_{12}}{c_{13}}
$$

Thus, the equation of heat wave in transversely isotropic media becomes

$$
\nabla_{t t} \theta+\frac{1}{\tau} \nabla_{t} \theta=c^{2} \beta_{11}\left(\partial_{x}^{2}+\partial_{y}^{2}+\frac{\beta_{33}}{\beta_{11}} \partial_{z}^{2}\right) \theta
$$

where

$$
c=\sqrt{\frac{1}{\left(v+\frac{a_{1}^{*} a_{1}^{*}}{\lambda_{1}}+\frac{a_{2}^{*} a_{2}^{*}}{\lambda_{2}}+\frac{a_{3}^{*} a_{3}^{*}}{\lambda_{3}}+\frac{a_{4}^{*} a_{4}^{*}}{\lambda_{4}}\right) T \tau}}
$$

Now, we discuss the propagation properties of heat wave in transversely isotropic media along $\mathrm{z}$ direction. At this moment, Equation (39) becomes

$$
\nabla_{t t} \theta+\frac{1}{\tau} \nabla_{t} \theta=c^{2} \beta_{33} \frac{\partial^{2}}{\partial^{2} z} \theta
$$

The plane wave solution of Equation (41) is the following

$$
\theta=\theta_{0} \exp [i(\omega t-\overline{k z})]
$$

Substituting the above into Equation (41), we have

$$
\omega^{2}-i \omega \frac{1}{\tau}=\beta_{33} c^{2} \bar{k}^{2}
$$

where

$$
\bar{k}=k_{1}+i k_{2}
$$

By using Equation (44), Equation (43) becomes

$$
\omega^{2}-i \omega \frac{1}{\tau}=\beta_{33} c^{2}\left(k_{1}^{2}-k_{2}^{2}+2 i k_{1} k_{2}\right)
$$

Comparing the real and imaginary parts of Equation (45), we have

$$
\omega^{2}=\beta_{33} c^{2}\left(k_{1}^{2}-k_{2}^{2}\right), \omega=-2 k_{1} k_{2} \beta_{33} \tau c^{2}
$$

From the above, we have

$$
k_{2}^{4}+\frac{\omega^{2}}{\beta_{33} c^{2}} k_{2}^{2}-\left(\frac{1}{2 \tau \sqrt{\beta_{33}} c}\right)^{2} \frac{\omega^{2}}{\beta_{33} c^{2}}=0
$$

when $\frac{1}{2 \tau \omega} \gg 1$, we get

$$
k_{1} \approx \frac{\omega}{\sqrt{\beta_{33}} c}, \quad k_{2} \approx-\frac{\omega}{2 \tau \sqrt{\beta_{33}} c}
$$

Thus, the plane wave solution of Equation (41) can be written as

$$
\theta=\theta_{0} \exp \left(-\frac{1}{2 \tau \sqrt{\beta_{33}} c} z\right) \exp [i(\omega t-k z)]
$$

or

$$
\theta=\theta_{0} \exp \left(-\frac{1}{2 \tau} t\right) \exp [i(\omega t-k z)]
$$

It is seen from the above that heat wave is of dissipative properties, it will attenuate with the distance or time of wave propagation. A possible explanation for the results is that in an idealized solid, the thermal energy can be transported by quantized electronic excitations and by the quanta of lattice vibrations, these quanta undergo collisions of a dissipative nature, causing a thermal resistance in the medium.

Two extreme cases are the following

1) $\tau \rightarrow \infty, \theta=\theta_{0} \exp [i(\omega t-k z)], c \rightarrow 0$

2) $\tau \rightarrow 0, \theta \rightarrow 0, c \rightarrow \infty$

Case 1 shows that no heat wave propagates when the dissipation coefficients in media is large enough. All thermal disturbances will be totally absorbed by the media.

Case 2 shows that heat wave propagates at an infinite speed when no dissipation exists in media, which is just the classical result of thermo-elasticity based on the conventional heat conduction equation.

\section{Conclusions}

Based on the quasi-static approximation, the heat wave in anisotropic elastic media is studied by using the eigen theory. An new thermo-elastic model based on the second law of thermodynamics is induced, the uncoupled heat wave equation is deduced, the propagation speed of heat wave is obtained. The results show that the equation of heat wave is of the strongly dissipative properties, and when the dissipation coefficients is large enough, no heat wave propagates in media, it means that thermal disturbances will be totally absorbed by the media, but when no dissipation exists, heat wave will propagate at an infinite speed, which is certainly unacceptable in physics.

\section{References}

[1] H. Lord and Y. Shulman, "A Generalized Dynamical Theory of Thermo-Elasticity," Journal of the Mechanics 
and Physics, Vol. 15, No. 5, 1967, pp. 299-309.

[2] R. Dhaliwal and H. Sherief, "Generalized Thermoelasticity for Anisotropic Media," The Quarterly of Applied Mathematics, Vol. 33, Vol. 1, 1980, pp. 1-8.

[3] A. E. Green and K. E. Lindsay, "Thermoelasticity," Journal of Elasticity, Vol. 2, 1972, pp. 1-7.

[4] X. G. Tian, Y. P. Shen, et al., "A Direct Finite Element Method Study of Generalized Thermoelastic Problems," International Journal of Solids and Structures, Vol. 43, No. 7-8, 2006, pp. 2050-2063.

[5] T. C. Chen and C. I. Weng, "Generalized Coupled Transient Thermoelastic Plane Problems by Laplace Transform/Finite Element Method," Journal of Applied Mechanics, Vol. 55, No. 2, 1988, pp. 377-382.

[6] J. H. Prevost and D. Tao, "Finite Element Analysis of
Dynamic Coupled Thermoelasticity Problems with Relaxation Times," Journal of the Mechanics and Physics, Vol. 50, 1983, pp. 817-822.

[7] S. H. Guo, "An Eigen Theory of Rheology for Complex Media," Acta Mechanica, Vol. 182, 2007, pp. 985-992.

[8] S. H. Guo, "An Eigen Theory of Electromagnetic Waves Based on the Standard Spaces," International Journal of Engineering Science, Vol. 47, 2009, pp. 405-412.

[9] S. H. Guo, "An Eigen Theory of Waves in Piezoelectric Solids," Acta Mechanica Sinica, Vol. 26, No. 2, 2010, pp. 241-246.

[10] S. H. Guo, "An Eigen Theory of Electro-Magnetic Acoustic Waves in Magnetoelectroelastic Media," Acta Mechanica, Vol. 211, No. 1-2, 2010, pp. 173-180. 